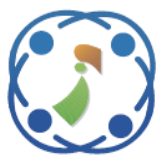

\title{
Seizure Prediction Based on Convolution Neural Network with Subnet Loss Function
}

\author{
Anuradha Yarlagadda ${ }^{1 *}$ \\ Ramakrishna Murty Malla ${ }^{2}$ \\ Hyma Janapana ${ }^{3}$ \\ ${ }^{I}$ Department of Computer Science and Engineering, \\ Gayatri Vidhya Parishad College of Engineering (A), Visakapatnam, India \\ ${ }^{2}$ Department of Computer Science and Engineering, ANITS, Visakapatnam, India \\ ${ }^{3}$ Department of Computer Science and Engineering, GITAM University, Visakapatnam, India \\ * Corresponding author's Email: anuradhayarlagaddag@gvpce.ac.in
}

\begin{abstract}
Electroencephalogram (EEG) signals of patients are applying for the classification and prediction of seizures. Accurate prediction of seizures helps the doctor to decide on the patients' treatment. Various existing models were applied for the prediction of seizures based on feature extraction and deep learning techniques and it also has the limitations of lower efficiency in feature analysis and overfitting problems in deep learning techniques. In this research, the Convolutional Neural Network $(\mathrm{CNN})$ with subnet loss function is proposed for the prediction of seizures. The subnet loss function is applied in CNN model to update the network model with suitable loss value. The subnet loss function is based on probabilistic distance between average embedding in the network. The CNN-VGG16 architecture is applied for the prediction of seizures due to model has higher efficiency in handling the features. The three-loss function is used, the first loss function is used for the feature analysis, the second loss function is applied for the feature map and the third loss function is applied for retraining useful information. To evaluate the performance of the proposed model, two datasets such as iEEG and rs-fMRI datasets were used. The proposed CNN-SLF has the advantage of retrain useful information to provide effective performance. The result shows that the proposed CNNSLF model has $99.2 \%$ accuracy and the existing Long Short Term Memory (LSTM) with deep learning features has the accuracy of $98 \%$.
\end{abstract}

Keywords: Convolutional neural network, Electroencephalogram, Long short term memory, Subnet loss function, VGG16 architecture.

\begin{tabular}{|c|l|}
\hline$A$ & Loss discriminator \\
\hline$a, b$ & Cycle Consistency \\
\hline$B$ & Dataset \\
\hline$D_{B}$ & Dataset discriminator \\
\hline$G_{f}$ & $\begin{array}{l}\text { Forward generator } \\
\text { network }\end{array}$ \\
\hline$G_{r}$ & $\begin{array}{l}\text { Reverse generator } \\
\text { network }\end{array}$ \\
\hline$H()$ & $\begin{array}{l}\text { Softmax cross-entropy } \\
\text { loss function }\end{array}$ \\
\hline$k$ & Kernel \\
\hline$p$ & Predicted output \\
\hline$r$ & True label \\
\hline$x$ & Input data \\
\hline$y$ & Label of data \\
\hline
\end{tabular}

\section{Introduction}

Electrical activity of Epilepsy patients' brains has been considered to provide significant information towards seizures onset and various methods were applied to isolate rhythmic changes and preictal EEG patterns to detect an incoming seizure [1]. In the biomedicine field, the seizure prediction study plays an important role to help orthobiosis to select the proper treatment for the patient [2]. Brain electrical 
activity were monitored based on the Electroencephalogram (EEG) signals and EEG electrodes were placed on the patients' scalp to record these signal for electrodes implantation called intracranial EEG (iEEG) signal [3]. Epileptologists experts are required to analyze the vast amount of data for epilepsy diagnosis and EEG visual analysis requires several hours [4]. Recently, many researches were carried out to detect non-epileptic and epileptic signals for the signal classification. Machine learning techniques were widely applied in the non-epileptic and epileptic signal classification. Generally, epilepsy data were applied in a small amount for training a classifier due to the presence of less number of seizures [5].

In pre-processing, discrete wavelets transform, continuous wavelet transform, and empirical mode decomposition were applied in many studies. For feature selection, Principle Component Analysis (PCA) were applied in few studies. After feature selection, classification is required for epileptic signal classification and Support Vector Machine (SVM) was applied in various studies for the classification [6, 7]. In the continuous recorded EEG data, non-seizures and seizures statistical features were extracted to improve the prediction accuracy. Commonly used brain imaging tools such as Magnetic Encephalography (MEG), Positron Emission Tomography (PET), and functional Magnetic Resonance Imaging (fMRI) were widely used and are limited by suitability for long-term monitoring, mobility, cost, low spatial and temporal resolution. Various researches show that EEG signals provide useful information in clinical contexts for diagnosis $[8,9]$. Recently, deep learning methods were used for effective image and signal classification [10]. In this research, the CNN-VGG16 with subnet loss function is proposed to improve the efficiency of seizure prediction. The EEG and MRI datasets were used to evaluate the performance of the proposed model. The sub-net loss function update the network loss value based on distance between probabilistic distribution embedding. The three loss value is measured from the network and overall loss function is updated based on sum of three loss value. The bi-directional analysis in loss function helps to provide the suitable loss value and Middle layer in sub-net preserve the useful features.

This paper is organized as follows: A review of recent methods in seizure prediction is provided in Section 2 and the explanation of CNN with subnet loss function is provided in Section 3. The result of the proposed model in EEG and MRI data is given in Section 4 and the conclusion is given in Section 5.

\section{Literature review}

Various existing models were applied for the prediction of seizures based on the EEG and fMRI data. Some of the notable researches in seizure prediction in EEG data were reviewed in this section.

Hosseini [11] developed a non-invasive and invasive method for monitoring, evaluating and regulation of epileptic brain. Support Vector Machine (SVM) and Convolution Neural Network (CNN) based feature extraction model was proposed. The developed model was evaluated in the fMRI and EEG dataset to analyze the efficiency. The CNN-SVM method has a higher performance than existing methods in EEG signals. The SVM has a lower capacity in handling the large dataset and the CNN model has the overfitting problem.

Khan [12] applied a convolution filter on the wavelet transformation of the EEG signal for learning quantitative signature for each period. The optimal seizure prediction horizon was learned from the prior assumption. The developed model was evaluated on a large dataset and shows that model has higher efficiency. The developed model has significantly higher efficiency than existing methods and it has a lower efficiency in analyzing the relationship between the features.

Zhang [13] developed a two-step feature selection model for ranking and selection of features in a patient-specific manner. Second-order Kalman filters further process the selected features and apply them as input to the linear SVM model. The developed algorithm was tested on the intra-cranial EEG (iEEG) and scalp EEG (sEEG) to analyze the performance. The developed model has a higher performance in seizure detection. The developed model has a lower efficiency in long recording datasets and feature analysis.

George [14] proposed Artificial Neural Network (ANN), Particle Swarm Optimization (PSO), Tunable-Q Wavelet Transform (TQWT) for the prediction of an epileptic seizure. Various non-linear features were evaluated to analyze the performance of the developed model. The developed model performance was evaluated based on two EEG datasets and shows that the developed model has a higher efficiency in seizure prediction. The PSO method has the limitations of poor convergence and the overfitting problem in ANN is required to be reduced.

Segundo [15] proposed deep learning architecture of two convolution layers for feature extraction and classification was performed based on a three-fully connected network. Empirical mode decomposition, wavelet and Fourier were applied to generate the 
input to the deep learning network. Model performance was evaluated on two public datasets and shows higher efficiency than existing methods in seizure prediction. The $\mathrm{CNN}$ model has the overfitting problem in the prediction process and this can be overcome feature selection in the training process.

Santoso [16] applied CNN model for multisegment of EEG signal to classify epileptic EEG signal. The ensemble combination is added in CNN model and each channel of signal is partitioned without overlapping to develop multi-segment of EEG signal. Short time Fourier transform value is used to convert the spectrogram image. The spectrogram image is applied as input to the CNN model for training and testing. The $\mathrm{CNN}$ model has overfitting problem and higher error rate in classification.

Ali and Elfattah [17] applied Lion Optimization Algorithm (LOA) and Support Vector Machine (SVM) for epileptic seizure detection to find the optimum parameter for SVM. The DWT method is applied to partition the EEG signal in five combinations. The developed method has higher performance in the seizure detection method. The LOA method has local optima problem and SVM method has imbalance dataset problem.

Wijaya [18] applied Logistic Regression for feature selection and two stage detection in channel instantiation method. The Linear Discriminative Analysis is applied in first stage and combination of K Nearest Neighbor and Gradient Boosting Tree is applied in second stage. The developed method has higher performance in seizure classification compared to existing method. The KNN model performance depends on the quality of data and sensitive to irrelevant features in the input dataset.

Das [19] applied various feature extraction methods such as target point selection, lower threshold and current maxima, and pattern matching methods such as domain and segment matching for seizure classification. Physiological field features, energy, power, homogeneity, and maxima features were used for proposed method. The developed method shows the considerable performance in the classification. Feature extraction methods creates overfitting problem in training the classifier.

Usman [20] applied empirical mode decomposition based on baseband filtering for noise removal in EEG signal. Generative Adversarial Network is applied for mitigation of synthetic preictal segment to solve imbalance data problem. The three layer CNN based model is applied to extract features from preprocessed EEG signal and combine with handcrafted features to improve the seizure

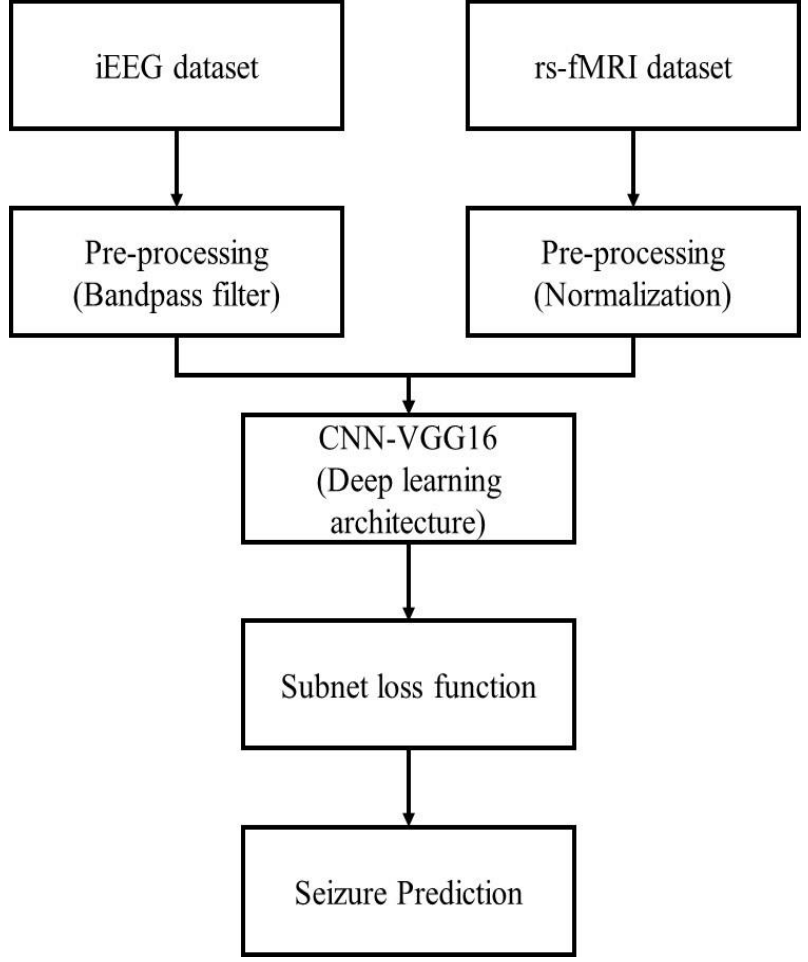

Figure. 1 The block diagram of CNN-VGG16 architecture with a subnet loss function

classification. Overfitting problem occurs in the $\mathrm{CNN}$ model due to presence of more features.

\section{Method}

Existing methods applied feature extraction and deep learning features for the prediction of seizures. In this research, the CNN-VGG16 with subnet loss function is proposed to improve the efficiency of seizure prediction. The iEEG and rs-fMRI datasets were applied to evaluate the performance of the proposed model. In pre-processing, the bandpass filter is applied for EEG data and normalization is applied for MRI data. The overview of the proposed CNN-SLF model is shown in Fig. 1.

\subsection{Pre-processing of EEG data}

The bandpass filter with 0.1 to $44 \mathrm{~Hz}$ cut-off frequency was applied in the EEG signal in the preprocessing stage. Short-Time Fourier Transform (STFT) was applied in the EEG signals to convert the EEG signals in 2-dimensional input due to $\mathrm{CNN}$ requires the $2 \mathrm{D}$ input. The STFT was set with the following specifications as 256 FFT length, $75 \%$ overlap, and 63 lengths of Kaiser window with a shape of 1 parameter. Vertically concatenate the 19 channels spectrogram to create final image. An overlap technique is applied to provide balanced samples or images to overcome the imbalance dataset problem in CNN. 


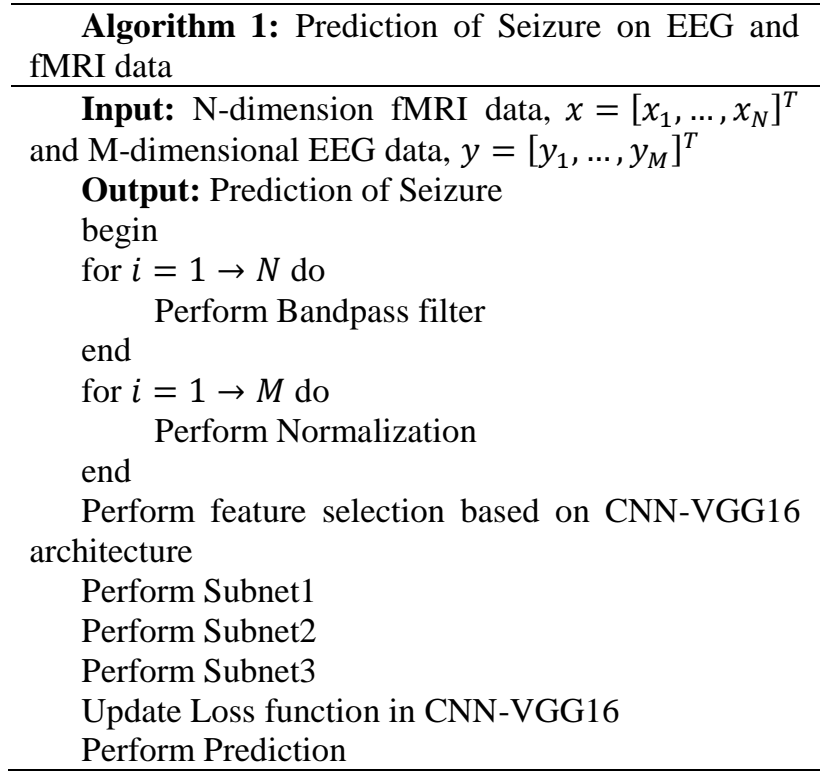

\subsection{Pre-processing of fMRI data}

The DPARSF toolbox (http://www.restfmri.net) is applied in this study for pre-processing fMRI data. After performing realignment, slice-timing, and first 10 images, Gaussian kernel of FWHM $=4$ is applied to smooth the data and Echo-Planar Imaging (EPI) is applied for normalization in a voxel size of $3 \times 3 \times 3$ $\mathrm{mm}$ in MNI space. All volumes were filtered and detrended with a Bandwidth of $0.01-0.08 \mathrm{~Hz}$.

\subsection{Convolution neural network}

After pre-processing of EEG signal data, spectrum images of time-frequency sets are generated. The transfer learning method is followed in training the deep CNN model due to the long time for training CNN and insufficient labeled data in practical applications. Transfer learning with a limited amount of labeled data improves the CNN training efficiency. Several previous studies [21 - 23] show that transfer pre-trained DNN parameters and partial structure in the source domain to the target domain network reduces the need for labeled data in the target domain and training time. CNN front-layers low-level features are common and later-layer highlevel features are specific for the task. CNN frontlayers are considered as a universal feature extractor.
Transfer CNN framework is applied to classify Spectrum images and EEG signals. Target $\mathrm{CNN}$ and the Pre-trained VGG-16 CNN model are applied in the proposed framework. The pre-trained VGG-16 CNN model is applied for common image classification tasks and the target $\mathrm{CNN}$ model is applied for the epilepsy classification. The target CNN, pre-trained VGG-16 CNN and the training process of the proposed framework are given as follows and the architecture is shown in Fig. 2.

\subsection{Pre-trained VGG-16 CNN}

Oxford Visual Geometry Group developed VGG$16 \mathrm{CNN}$ model with 16 layers and achieved significant performance in various image classification tasks. The VGG-16 applied small-sized filters instead of large-sized convolution filters and improves the network depth [24 - 25]. CNN with small filters provides more benefits to increases the classification accuracy. For the image classification task, low-level features extraction based on pretrained $\mathrm{CNN}$ and VGG-16 CNN of pre-trained ImageNet dataset.

\subsection{Target CNN}

Target CNN and pre-trained VGG-16 model has the almost same structure and a softmax output layer is present in target CNN [26]. An output layer of the new softmax is added instead of the original output layer and the number of neurons is the same as the class of the data. For EEG signal classification, the softmax classifier is used. Pre-trained CNN model structures, parameters, and hyper-parameters are applied to the target CNN model to increases the efficiency. Target $\mathrm{CNN}$ is fine-tuned for specific EEG classification tasks without train the whole network. The target $\mathrm{CNN}$ fine-tuning of the loss function is softmax cross-entropy, as given in Eq. (1).

$$
H(r, p)=-\sum_{i} r_{i} \log \left(p_{i}\right)
$$

where output probability is denoted as $p$, if predicted output and true label are the same, then $r$ is 1 , or $r$ is 0 .
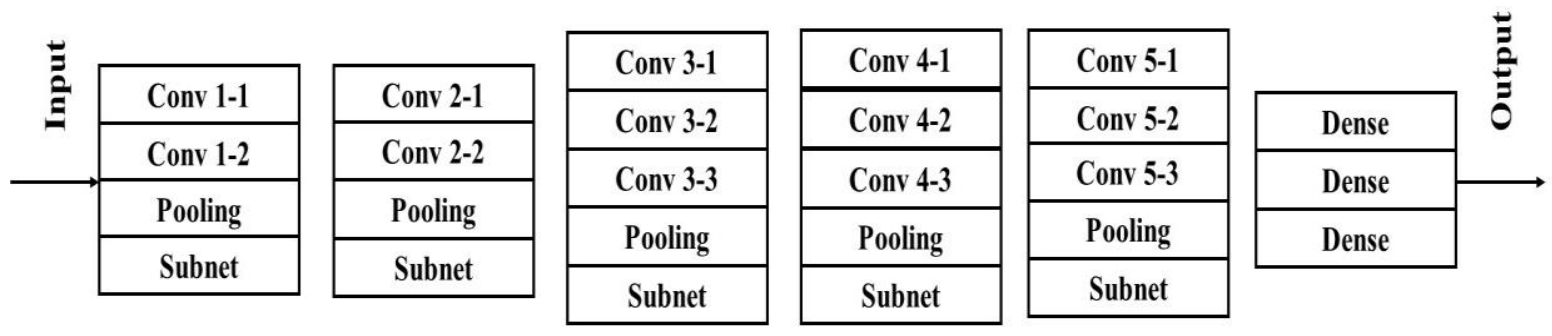

Figure. 2 The architecture of CNN-VGG16 with a subnet loss function 


\subsection{Training procedure}

Training and testing data is divided from timefrequency after a successful design of target $\mathrm{CNN}$ and pre-trained VGG-16 CNN [27]. The target CNN is fine-tuning with training-dataset, and the testing dataset is used to evaluate the performance of the model. ImageNet dataset and target dataset is not similar for pre-training, this requires more layers for the target $\mathrm{CNN}$ to be fine-tuned. Target $\mathrm{CNN}$ of layer 1 to layer 11 of the front layers are frozen and later layers after 11 are set to be trainable, target dataset is used to fine-tune these layers.

\subsection{Subnet loss function}

The VGG-16 structure and subnet1 structure are similar and this ensures the information is not lost excessively in $A=A^{\prime}$ constraint. Two data conversion models are no longer trained based on two independent networks. Two models are shared for parameters and the model has capable of bidirectional input and output.

Two loss functions are fine-tuned based on the task, as given in Eqs. (2) and (3).

$$
\begin{gathered}
\mathcal{L}_{c y c}\left(G_{f}, G_{r}\right)=\mathbb{E}_{\alpha \sim \operatorname{pdata}(a)}\left[\| G_{r}\left(G_{f}(\alpha)\right)-\right. \\
\left.\alpha \|_{1}^{2}\right]+\mathbb{E}_{b \sim \operatorname{pdata}(b)}\left[|| G_{f}-\left(G_{r}(b)\right)-\left.b\right|_{1} ^{2}\right. \\
\mathcal{L}_{V D D 16}\left(G_{f}, D_{B}, A, B\right)=\mathbb{E}_{b \sim \operatorname{data}(b)}\left[D_{B}(b)\right]+ \\
\mathbb{E}_{\alpha \sim \operatorname{pdata}(\alpha)}\left[1-D_{B}\left(G_{f}(\alpha)\right)\right]
\end{gathered}
$$

The forward input model is used to obtain the generator network $G_{f}$ and reverse input comes from $G_{r}$. Cycle consistency $a \rightarrow G_{f}(a) \rightarrow G_{r}\left(G_{f}(a)\right) \approx$ $a$ is for each signal $a$ from domain $A$ and similarly $b$. $\log$ operations are not used by $\mathcal{L}_{V G G 16}\left(G_{f}, D_{B}, A, B\right)$ that provide more stable training. The dataset $B$ discriminator is $D_{B}$ and discriminator $D_{A}$ that is $\mathcal{L}_{V G G 16}\left(G_{r}, D_{A}, B, A\right)$. The subnet1 loss function is given in Eqs. (4) and (5).

$$
\begin{gathered}
\mathcal{L}_{s n 1}=\mathcal{L}_{c y c}\left(G_{f}, G_{r}\right)+\mathcal{L}_{V G G 16}\left(G_{f}, D_{B}, A, B\right)+ \\
\mathcal{L}_{V G G 16}\left(G_{f}, D_{B}, A, B\right) \\
G_{f}^{*}, G_{r}^{*}=\min _{G_{f}, G_{r} D_{A}, D_{B}}\left(\mathcal{L}_{s n 1}\right)
\end{gathered}
$$

The subnet3 is designed to retain useful information and Middle Content Layer features is useful information in the EEG signal. Three feature map output is present for dataset $A, 1 \rightarrow 2 \rightarrow 3$ : $\varphi 1(a) ; 6 \rightarrow 7 \rightarrow 8: \varphi 2(b) ; 6 \rightarrow 7 \rightarrow 8:$ $\varphi 2(G f(a))$. According to $b, a, G_{f}(a)$, the loss function is derived as Eq. (6).

$$
\begin{aligned}
& \mathcal{L}\left(B_{1 \sim 5}, A\right)=\mathcal{L}_{M S E_{A}}\left(B_{1 \sim 5}, A\right)+ \\
& \mathcal{L}_{M K M M D_{A}}\left(B_{1 \sim 5}, A, B\right)=\mathbb{E}\left[|| \varphi_{1}(a)-\right. \\
& \left.\left.\varphi_{2}\left(G_{f}(a)\right)\right|_{1} ^{2}\right]+\mathbb{E}\left[\| \mathbb{E}_{p}\left[\phi_{k}\left(\varphi_{1}(a)\right)\right]-\right. \\
& \left.\mathbb{E}_{1}\left[\phi_{k}\left(\varphi_{2}(b)\right)\right] \mid l\right]
\end{aligned}
$$

Where mapping function of $\phi_{\kappa}(\cdot)$ is related to the kernel, and $\varphi_{1}(\cdot), \varphi_{2}(\cdot)$ are $B_{1}\left(B_{2}\left(B_{3}(\cdot\right.\right.$ ))), $B_{5}\left(B_{4}\left(B_{3}(\cdot)\right)\right)$, respectively. Two probability distribution $p$ and $q$ of the distance between the average embedding in Reproducing Kernel Hilbert Space (RKHS) with kernel $\kappa$ is the MK-MMD distance, as given in Eq. (7).

$$
\mathcal{L}_{\text {sn } 3}=\mathcal{L}_{\text {mid }}\left(B_{1 \sim 5}, A\right)+\mathcal{L}_{\text {mid }}\left(B_{1 \sim 5}, B\right)
$$

Block3 consists of BCG-removed and original signal similar features that retain better information. This model is an upstream task and there is no downstream task to provide good feature output for future downstream tasks.

The data denoising is more complex without the assistance of classification tasks. Training of the main network is assisted by multiple sub-networks that add more constraints to correct training direction. Each decomposed block provides specific functions in joint training to improve the overall network performance, as given in Eq. (8).

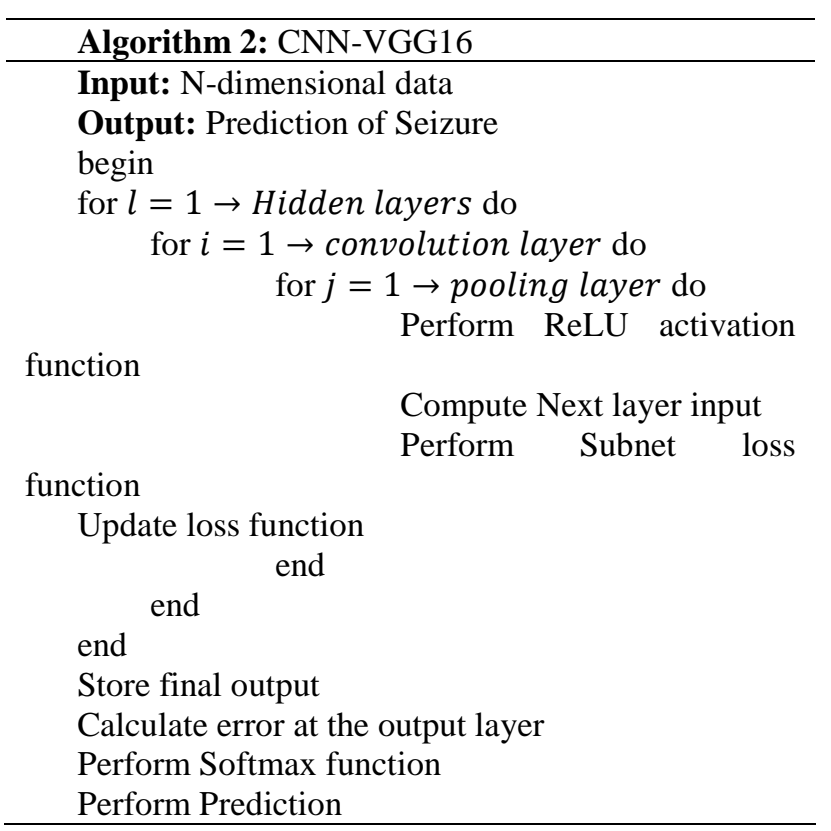




$$
\mathcal{L}_{\text {total }}=\lambda\left(\mathcal{L}_{s n 1}+\mathcal{L}_{s n 2}+\mathcal{L}_{s n 3}\right)
$$

Where weight assignment vector is denoted as $\lambda$.

\section{Result}

Early epilepsy prediction helps the doctor to decide the treatment for patients and treat the patient effectively. Various existing models were applied for the prediction of epilepsy and existing models have the limitations of overfitting problems. In this research, the subnet loss function is applied in the CNN-VGG16 network to improve the efficiency of epilepsy detection. The proposed model is applied in EEG and fMRI data to evaluate the model. This section provides the details and results of the proposed model.

Dataset: Temporal and extratemporal epilepsy of eight epileptics in the iEEG dataset were studied. A sampling rate in a range of 500 to $5000 \mathrm{~Hz}$ of preictal and non-prerictal segments were used for training and testing. The window size is set as $1 \mathrm{~s}$ and a total of 1650 segments are used. The rs-fMRI data of 160 scans were collected from Henry Ford Hospital in Detroit, MI. The iEEG [11] and rs-fMRI [11] datasets are used to test the performance of proposed and existing methods in prediction.

System Configuration: The proposed model is executed in the system of Intel i9 process, $128 \mathrm{~GB}$ of RAM, 22 GB graphics card and 3 TB hard disk. The MATLAB R2020a tool were used to evaluate the proposed model.

Metrics: The metrics such as Accuracy, sensitivity, and specificity were used to test the proposed model efficiency.

Parameter Settings: The learning rate is set as 0.01 , dropout rate is set as 0.001 , kernel size is set as 3 and 'ReLU' activation function is used.

\subsection{Quantitative analysis}

The proposed CNN-SLF model is evaluated on the EEG and fMRI data to analyze the effectiveness.

The proposed CNN-SLF model is evaluated on EEG and fMRI data to analyze the effectiveness, as shown in Table 1. The proposed CNN-SLF model has

Table 1. Performance analysis of the proposed model

\begin{tabular}{|c|c|c|c|}
\hline $\begin{array}{c}\text { Classifier } \\
\text { S }\end{array}$ & $\begin{array}{c}\text { Accuracy } \\
(\boldsymbol{\%})\end{array}$ & $\begin{array}{c}\text { Sensitivity } \\
(\boldsymbol{\%})\end{array}$ & $\begin{array}{c}\text { Specificity } \\
(\boldsymbol{\%})\end{array}$ \\
\hline SVM & 83.5 & 85.1 & 84.2 \\
\hline LSTM & 90.3 & 91.4 & 91.2 \\
\hline CNN & 93.8 & 94.2 & 94.3 \\
\hline CNN- & & & \\
VDD16 & 95.6 & 96.4 & 97.4 \\
\hline CNN-SLF & 99.2 & 97.4 & 97.7 \\
\hline
\end{tabular}

a higher performance in terms of accuracy, sensitivity and specificity compared to the existing model. The subnet activation function analysis the data in threelayer and effectively retrain the information that improves the proposed model performance. The proposed CNN-SLF model has $99.2 \%$ accuracy and the existing CNN-VDD16 has $95.6 \%$ accuracy.

The accuracy of the proposed CNN-SLF model and the existing model is compared in Fig. 3. The result shows that the proposed CNN-SLF model has higher accuracy compared to existing models such as SVM, LSTM, CNN, and CNN-VDD16. The proposed CNN-SLF model has the advantage of applying a subnet activation function that effectively analysis the input data. The proposed CNN-SLF method has the accuracy of $99.2 \%$ accuracy and the existing CNN has $93.8 \%$ accuracy. The SVM has a lower performance due to the model has lower efficiency in handling the various feature value.

The sensitivity and specificity of the proposed CNN-SLF model are compared with the existing model in Fig. 4. The result shows that the proposed CNN-SLF model has higher performance compared to existing models. The subnet activation functions effectively analyze the input data and retrain the important information that helps to improve the

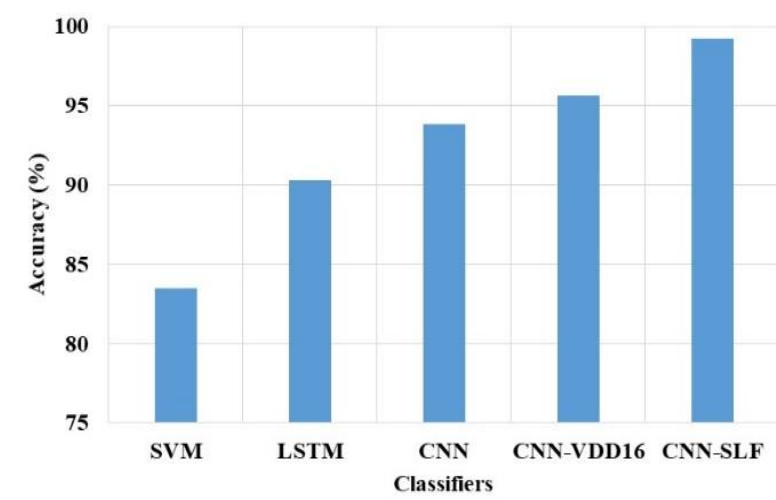

Figure. 3 Accuracy of the proposed CNN-SLF model

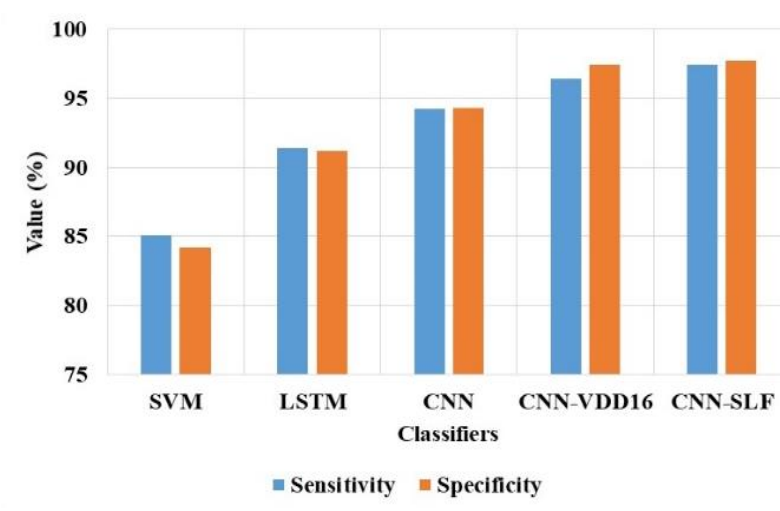

Figure. 4 Sensitivity and specificity of the proposed CNN-SLF model 
efficiency of the analysis. The CNN-VDD16 model has the limitation of loss of important information in feature analysis. The proposed CNN-SLF model has a sensitivity of $97.4 \%$ and existing CNN-VDD16 has $96.4 \%$ accuracy.

\subsection{Comparative analysis}

The proposed CNN-SLF model is compared with existing research [11-15] in seizure prediction.

The proposed and existing methods are tested for binary-class classification in EEG and fMRI dataset. The CNN [12], SVM [13], and CNN [15] methods were tested on EEG dataset and not for fMRI dataset. The proposed CNN-SLF model is compared with existing methods in seizure prediction is shown in Table 2. The result shows that the proposed CNNSLF model has higher performance compared to existing methods.

The proposed and existing methods in seizure prediction were compared in same environment and same datasets. The CNN [12] and SVM [13] method is evaluated on the EEG data and other compared techniques are evaluated on EEG and fMRI data. The proposed CNN-SLF model has the advantage of retrain important information. The proposed $\mathrm{CNN}$ SLF model has the accuracy of $99.2 \%$ and the existing SVM [11] model has $98 \%$ accuracy.

The proposed CNN-SLF model is compared with existing models in seizure prediction as shown in Fig. 5. The result shows that the proposed CNN-SLF model has higher performance compared to existing methods. The sub-net loss function retains the useful features in the network and update the loss function suitable for multi-class classification. The loss function update helps to overcome the limitation of overfitting due to relevant feature selection. The existing CNN model $[12,15]$ has overfitting problem and PSO [14] method easily trap into local optima. The proposed CNN-SLF model has the advantages of applying subnet activation that retrain important information. The proposed CNN-SLF model has the accuracy of $99.2 \%$ and existing $97.4 \%$ accuracy.

Table 2. Comparative analysis of proposed CNN-SLF model

\begin{tabular}{|c|c|c|c|c|c|}
\hline Classifiers & Feature Extraction & Data & $\begin{array}{c}\text { Accuracy } \\
(\mathbf{\%})\end{array}$ & $\begin{array}{c}\text { Sensitivity } \\
(\mathbf{\%})\end{array}$ & $\begin{array}{c}\text { Specificity } \\
(\mathbf{\%})\end{array}$ \\
\hline LSTM + SVM [11] & $\begin{array}{c}\text { Deep Learning + Hand Crafted } \\
\text { (Time, Freq., Wavelet, Filtering) }\end{array}$ & EEG + fMRI & 98 & 96 & 97 \\
\hline CNN [12] & Hand Crafted (Wavelet) & EEG & 90 & 87 & 89 \\
\hline SVM [13] & Hand Crafted (Spectral Power) & EEG & 98 & 98 & 97 \\
\hline ANN + PSO [14] & Wavelet Transform & EEG + fMRI & 97.4 & 97.2 & 97.1 \\
\hline CNN [15] & Empirical Mode Decomposition & EEG & 99.5 & - & - \\
\hline $\begin{array}{c}\text { Pattern Matching } \\
{[19]}\end{array}$ & $\begin{array}{c}\text { Physiological field features, } \\
\text { energy, power, homogeneity, and } \\
\text { maxima features }\end{array}$ & EEG + fMRI & 98.2 & 97.3 & 97.5 \\
\hline \multirow{2}{*}{ GAN + CNN [20] } & $\begin{array}{c}\text { CNN extracted features + } \\
\text { Handcrafted features (statistical } \\
\text { and Entropy features) }\end{array}$ & EEG + fMRI & 98.7 & 97.9 & 97.7 \\
\hline CNN-SLF & Deep Learning & EEG & 99.8 & 99.7 & 99.6 \\
\cline { 2 - 6 } & EEG + fMRI & 99.2 & 97.4 & 97.7 \\
\hline
\end{tabular}

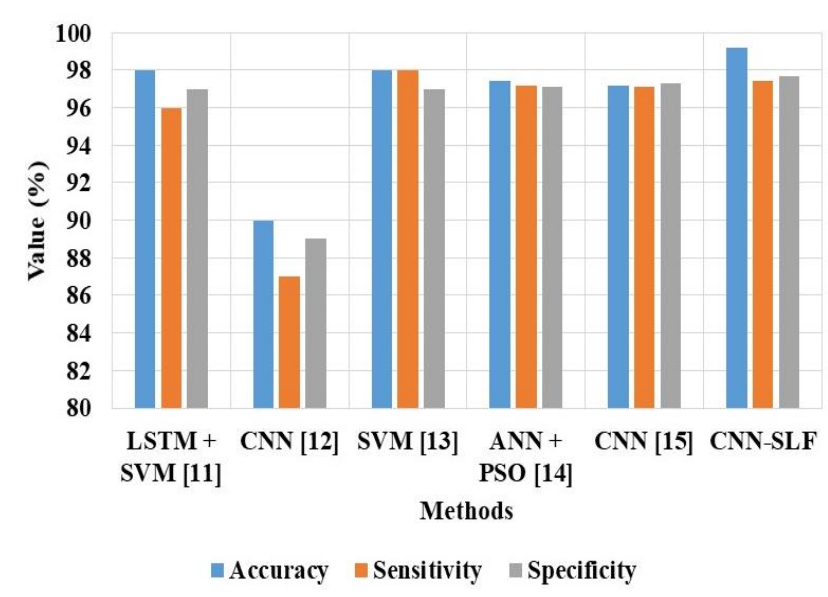

Figure. 5 Comparative analysis of proposed CNN-SLF and existing model 


\section{Conclusion}

Accurate and early prediction of seizures helps the doctors to decide on the treatment of a patient. Existing CNN, CNN-VGG16 models applied deep learning features and feature extraction techniques for the prediction of seizures and it has the limitations of overfitting problems and lower efficiency in feature analysis. In this paper, the CNN-VGG16 with subnet loss function is applied to improve the efficiency of seizure prediction. The subnet loss function is applied to improve the learning performance of the CNN model. The subnet loss function has three phases, the first phase involves analysis of the features, the second phase involves feature mapping, and the third phase involves in retrain useful information. The datasets such as iEEG and rs-fMRI were used to evaluate the performance of the proposed model. The proposed model has the advantage of retraining the important information in the training of the CNN-VGG16 architecture. The result shows that the proposed CNN-SLF method has $99.2 \%$ accuracy and the existing LSTM with deep learning features has $98 \%$ accuracy. The future direction of this method involves applying the attention layer in the deep learning method for medical image classification.

\section{Conflicts of Interest}

The authors declare no conflict of interest.

\section{Author Contributions}

The paper background work, conceptualization, methodology, dataset collection, implementation, result analysis and comparison, preparing and editing draft, visualization, supervision, review of work and project administration, have been done by first author.

\section{References}

[1] K. M. Tsiouris, V. C. Pezoulas, M. Zervakis, S. Konitsiotis, D. D. Koutsouris, and D. I. Fotiadis, "A long short-term memory deep learning network for the prediction of epileptic seizures using EEG signals", Computers in Biology and Medicine, Vol. 99, pp. 24-37, 2018.

[2] Y. Zhang, Y. Guo, P. Yang, W. Chen, and B. Lo, "Epilepsy seizure prediction on eeg using common spatial pattern and convolutional neural network", IEEE Journal of Biomedical and Health Informatics, Vol. 24, No. 2, pp. 465474, 2019.

[3] S. M. Usman, S. Khalid, and M. H. Aslam, "Epileptic seizures prediction using deep learning techniques", IEEE Access, Vol. 8, pp. 39998-40007, 2020.

[4] K. D. Tzimourta, A. T. Tzallas, N. Giannakeas, L. G. Astrakas, D. G. Tsalikakis, P. Angelidis, and M. G. Tsipouras, "A robust methodology for classification of epileptic seizures in EEG signals", Health and Technology, Vol. 9, No. 2, pp. 135-142, 2019.

[5] I. Ullah, M. Hussain, and H. Aboalsamh, "An automated system for epilepsy detection using EEG brain signals based on deep learning approach", Expert Systems with Applications, Vol. 107, pp. 61-71, 2018.

[6] S. M. Usman, S. Khalid, R. Akhtar, Z. Bortolotto, Z. Bashir, and H. Qiu, "Using scalp EEG and intracranial EEG signals for predicting epileptic seizures: Review of available methodologies", Seizure, Vol. 71, pp. 258-269, 2019.

[7] H. A. Hadeethi, S. Abdulla, M. Diykh, R. C. Deo, and J. H. Green, "Adaptive boost LS-SVM classification approach for time-series signal classification in epileptic seizure diagnosis applications", Expert Systems with Applications, Vol. 161, p. 113676, 2020.

[8] H. Zhang, J. Su, Q. Wang, Y. Liu, L. Good, and J. M. Pascual, "Predicting seizure by modeling synaptic plasticity based on EEG signals-a case study of inherited epilepsy", Communications in Nonlinear Science and Numerical Simulation, Vol. 56, pp. 330-343, 2018.

[9] M. Savadkoohi, T. Oladunni, and L. Thompson, "A machine learning approach to epileptic seizure prediction using Electroencephalogram (EEG) Signal", Biocybernetics and Biomedical Engineering, Vol. 40, No. 3, pp. 1328-1341, 2020.

[10] Z. Wei, J. Zou, J. Zhang, and J. Xu, "Automatic epileptic EEG detection using convolutional neural network with improvements in timedomain”, Biomedical Signal Processing and Control, Vol. 53, p. 101551, 2019.

[11] M. P. Hosseini, T. X. Tran, D. Pompili, K. Elisevich, and H. S. Zadeh, "Multimodal data analysis of epileptic EEG and rs-fMRI via deep learning and edge computing", Artificial Intelligence in Medicine, Vol. 104, p. 101813, 2020.

[12] H. Khan, L. Marcuse, M. Fields, K. Swann, and B. Yener, "Focal onset seizure prediction using convolutional networks", IEEE Transactions on Biomedical Engineering, Vol. 65, No. 9, pp. 2109-2118, 2017.

[13] Z. Zhang and K. K. Parhi, "Low-complexity seizure prediction from iEEG/sEEG using spectral power and ratios of spectral power", 
IEEE Transactions on Biomedical Circuits and Systems, Vol. 10, No. 3, pp. 693-706, 2015.

[14] S. T. George, M. S. P. Subathra, N. J. Sairamya, L. Susmitha, and M. J. Premkumar, "Classification of epileptic EEG signals using PSO based artificial neural network and tunableQ wavelet transform", Biocybernetics and Biomedical Engineering, Vol. 40, No. 2, pp. 709-728, 2020.

[15] R. S. Segundo, M. G. Martín, L. F. D. Enriquez, and J. M. Pardo, "Classification of epileptic EEG recordings using signal transforms and convolutional neural networks", Computers in Biology and Medicine, Vol. 109, pp. 148-158, 2019.

[16] I. B. Santoso, Y. Adrianto, A. D. Sensusiati, D. P. Wulandari, and I. Purnama, "Epileptic EEG signal classification using convolutional neural network based on multi-segment of EEG signal", International Journal of Intelligent Engineering and Systems, Vol. 14, No. 3, pp. 160-176, 2021.

[17] M. A. S. Ali and M. A. Elfattah, "A hybrid model (SVM-LOA) for epileptic seizure detection in long-term EEG records using machine learning techniques", International Journal of Intelligent Engineering and Systems, Vol. 11, No. 5, pp. 162-172, 2018.

[18] A. Wijaya, T. B. Adji, and N. A. Setiawan, "Logistic Regression based Feature Selection and Two-Stage Detection for EEG based Motor Imagery Classification", International Journal of Intelligent Engineering and Systems, Vol. 14, No. 1, pp. 134-146, 2021.

[19] K. Das, D. Daschakladar, P. P. Roy, A. Chatterjee, and S. P. Saha, "Epileptic seizure prediction by the detection of seizure waveform from the pre-ictal phase of EEG signal", Biomedical Signal Processing and Control, Vol. 57, p. 101720, 2020.

[20] S. M. Usman, S. Khalid, and S. Bashir, "A deep learning based ensemble learning method for epileptic seizure prediction", Computers in Biology and Medicine, Vol. 136, p. 104710, 2021.

[21] S. Zhang, R. Wu, K. Xu, J. Wang, and W. Sun, "R-CNN-based ship detection from high resolution remote sensing imagery", Remote Sensing, Vol. 11, No. 6, p. 631, 2019.

[22] Z. Liu, J. Wu, L. Fu, Y. Majeed, Y. Feng, R. Li, and Y. Cui, "Improved kiwifruit detection using pre-trained VGG16 with RGB and NIR information fusion", IEEE Access, Vol. 8, pp. 2327-2336, 2019.

[23] M. C. Leong, D. K. Prasad, Y. T. Lee, and F. Lin, "Semi-CNN architecture for effective spatio- temporal learning in action recognition", Applied Sciences, Vol. 10, No. 2, p. 557, 2020.

[24] Z. Liu, J. Wu, L. Fu, Y. Majeed, Y. Feng, R. Li, and Y. Cui, "Improved kiwifruit detection using pre-trained VGG16 with RGB and NIR information fusion", IEEE Access, Vol. 8, pp. 2327-2336, 2019.

[25] D. Zhao, D. Zhu, J. Lu, Y. Luo, and G. Zhang, "Synthetic medical images using F\&BGAN for improved lung nodules classification by multiscale VGG16", Symmetry, Vol. 10, No. 10, p. 519, 2018.

[26] Z. H. Qu, J. Mei, L. Liu, and D. Y. Zhou, "Crack detection of concrete pavement with crossentropy loss function and improved VGG16 network model", IEEE Access, Vol. 8, pp. 54564-54573, 2020.

[27] C. Sitaula and M. B. Hossain, "Attention-based VGG-16 model for COVID-19 chest X-ray image classification", Applied Intelligence, Vol. 51, No. 5, pp. 2850-2863, 2021. 